\title{
Isolation protocol for patients with severe combined immune deficiency
}

\author{
Brenda Reid* and Sarah Courtney
}

\begin{abstract}
Infants with severe combined immune deficiency (SCID) typically present in the first few months of life with severe, recurrent, opportunistic infections, and without definitive treatment the condition is invariably fatal (Gaspar et al. 2013). Many centres believe that protective isolation is required for treatment of SCID patients once diagnosed, and the isolation protocol varies across institutions. This paper describes the isolation protocol for SCID requiring hematopoietic stem cell transplant (HSCT) that has been utilized at our institution for over the last 25 years. We believe that the profound immunodeficiency in SCID patients warrants a more restrictive treatment to limit the morbidity and mortality associated with HSCT. With this protocol, we have seen a very low infection rate in and have a very good survival rate for our SCID population.
\end{abstract}

\section{Introduction}

Infants with severe combined immune deficiency (SCID), who are not identified through newborn screening, typically present in the first few months of life with severe, recurrent, opportunistic infections, and without definitive treatment the condition is invariably fatal (Gaspar et al. 2013). The isolation protocol for SCID requiring hematopoietic stem cell transplant (HSCT) that has been utilized at our institution for more than the last 25 years involves treating patients in reverse isolation rooms with HEPA filtration and laminar air flow (LAF). Prior to entering the room, staff and visitors are required to wear clean hair covers, masks and shoe covers, sterile gowns and gloves, and perform a surgical hand wash (Patient Care Committee 2010). We also limit visitors to the patient room to parents and 1-2 designated alternative caregivers. Our isolation protocol for SCID is more restrictive than for other patients undergoing HSCT for other indications at our centre. The literature uses the terms reverse isolation and protective isolation interchangeably, but at our centre we use the term reverse isolation for SCID patients and use protective isolation for all other bone marrow transplant (BMT) patients. We believe that the profound immunodeficiency in SCID patients warrants a more restrictive treatment to limit the morbidity and mortality associated with HSCT. Our survival rate post HSCT for SCID patients with minimal infectious complications provides compelling support for this isolation protocol for SCID patients (Dalal et al. 2000; Grunebaum et al. 2006).

\section{Description of isolation protocol}

\section{LAF rooms}

The isolation rooms are set-up with a shared anteroom between 2 patient rooms. The anteroom is a dedicated clean space between the hallway and patient isolation room that is used to prepare for patient care. The anteroom is where staff and visitors prepare to
Nursing and Immunology/Allergy, The Hospital for Sick Children, Toronto, ON, Canada
Submitted 14 July 2015

Accepted 14 July 2015

Available online 14 July 2015

LymphoSign Journal 2:165-170 (2015)

dx.doi.org/10.14785/lpsn-2015-0011 
enter the patient room and it includes a medication fridge and other necessary supplies for each of the 2 patient rooms.

Each room is equipped with a dual control system and dual motors to enable continued operation of fans at all times. At no time is the fan to be shut off while a patient is in the room. The direction of airflow is from the HEPA filter panel at the head of the patient's bed to over the patient and out the door. Patients are required to move rooms after 3 months of continued use for scheduled maintenance (BMT Steering Committee 2013).

There is a policy regarding preventative maintenance of the rooms that involves: monthly visual/audio inspection of supply air/exhaust fans and servicing if necessary in each room; semi-annual millwright fan inspection; patient room electrical and mechanical maintenance after 3 months of continued use; quarterly air audits of rooms and anterooms; annual inspection of HEPA filters; and air particulate concentration analysis of empty rooms and replacement of HEPA filters as required in empty rooms. The records of maintenance are kept by the hospital Plant and Engineering department for a minimum of 10 years in accordance with our hospital's record retention policy, and the Unit Manager is responsible for keeping a log of scheduled maintenance for each room. Any substandard findings result in a report that is sent to the Unit Clinical Manager, the responsible Administrative Director, the Medical Director for BMT, and the manager of Plant and Operations (BMT Steering Committee 2014).

\section{Protocol for entering the room}

All staff and visitors entering the anteroom are instructed to wash their hands immediately upon entry. To prepare to enter the SCID patient's LAF room the caregiver then opens a sterile gown package and adds the appropriately sized sterile gloves to the pack, dons the hair cover, the mask, and lastly the shoe covers. Once ready, a thorough hand washing is done, and the gown is put on by holding it by the neck edges and tying the gown at the neck. Next, gloves are put on and pulled over the cuffs of the sterile gown. The caregiver can then tie the gown at the waist and enter the patient room. Hand washing using alcohol-based hand solution and changing of sterile gloves is done in the patient room if gloves become soiled such as after diaper changes, after the use of antineoplastic agents, and prior to sterile procedures. Caregivers may leave the LAF room once garbed to retrieve medication or supplies from the anteroom provided they wash their hands and change gloves prior to re-entry into the patient LAF room. Isolation protective gear is removed in the anteroom when ready to leave the room and hands are washed again after taking all protective gear off (BMT Steering Committee 2013).

Other considerations in the LAF room to maintain the isolation include caregivers avoiding touching themselves above their shoulders or below their waist and not sitting on the patient's bed. If items fall to the floor they should not be picked up, but rather should be swept into the anteroom either with the foot or picked up while exiting the patient room. If the item needs to go back into the LAF room it should be cleaned with the hospital-approved low level disinfectant. Diapers should be promptly removed from the patient LAF room and anteroom. We use dedicated medical equipment whenever possible, and if equipment needs to be shared between patients it is cleaned with the hospital-approved low level disinfectant (BMT Steering Committee 2013).

To minimize the risk of infection, we do not allow plants or fresh flowers on the BMT unit. Caregivers are discouraged from eating in the rooms and patient food that has been at room temperature for more than $2 \mathrm{~h}$ is discarded. Wet linens are removed immediately to prevent bacterial growth, the sinks in the reverse isolation room have their regulators turned off, we use sterile water for bathing, and sterile linens are used (BMT Steering Committee 2013).

\section{Visiting policy}

Our hospital has an open visiting policy for parents and legal guardians or other designated caregivers, and they may visit their child $24 \mathrm{~h}$ a day, 7 days a week. However, the unique infectious risks for SCID patients undergoing BMT have resulted in a more restrictive policy for other visitors. Siblings are not allowed to visit the unit (BMT Steering Committee 2013).

\section{Diagnostic tests and procedures}

Transporting patients in reverse isolation to areas of the hospital less familiar with the SCID population can be a stressor for patients/families and health care professionals. The reverse isolation policy and procedure is published in our hospital Policy and Procedure Database that is available for all staff to facilitate reverse 
isolation practices. Whenever possible, we perform diagnostic tests in the patient room i.e., portable $\mathrm{X}$-rays, ultrasounds, blood draws. If the patient needs to leave the room, the Manager of the unit notifies the Manager of the care area of the patient's isolation requirements prior to transport. Transportation of the patient is done with the patient in a respiratory hood connected either to an air or oxygen tank as indicated. If patients do not require oxygen, the airflow to the hood must be at $10 \mathrm{~L} / \mathrm{min}$. There is a designated hood used only in our unit for transportation of SCID patients, and it is cleaned with disinfectant after use. Transportation routes to procedural areas are chosen to minimize contact with other patients during transport and to avoid areas of the hospital under construction. If construction areas are unavoidable the patient must wear an N95 respirator mask during transport (BMT Steering Committee 2013).

\section{Surgical procedures}

Precautions are in place to maintain the reverse isolation status for SCID patients requiring the operating room services. Patients are booked as either the first or last case of the day. The isolation status is confirmed between the Unit manager and the OR manager prior to the procedure. The anesthesia assessment is ideally performed in the patient's room prior to going to the $\mathrm{OR}$, but it may be done in the OR in the OR positive pressure waiting room if it is unable to be completed on the ward. When the patient is ready for transfer to the OR, the patient is transferred in the same way as for other procedures with the patient's assigned nurse from the ward; if already assessed by anesthesia, the patient goes directly into the OR theatre. Post-surgery patients are transferred to the Post Anesthetic Recovery Unit (PACU). In the PACU patients are nursed in a positive pressure recover isolation room using the same isolation practices as on the unit (BMT Steering Committee 2013).

\section{Discussion}

There are documented benefits from sterile gloves and gown; clean hair covers, masks, and boots; surgical hand washing techniques; and LAF in reducing surgical infections (Hubble et al. 1996; American Association of Nurse Anesthetists 2013). Moreover, bacterial isolates are increased when LAF is interrupted in an OR by frequent opening of the doors (Smith et al. 2013). Maintaining sterility of the surgical environment is viewed as even more critical when you have a significantly immunocompromised patient involved (Castro 2008). The ward setting provides unique challenges regarding transmission of infections to SCID patients, as health care providers attend to multiple patients with the potential for transmitting virulent organisms that are "antimicrobial resistant" (Centers for Disease Control and Prevention [CDC] 2014). Vigilant reverse isolation of SCID patients in LAF can help minimize the risk of hospital-acquired infections. In our first publication of results for unrelated donor transplants for SCID, we reported only 5 episodes of bacterial sepsis in 16 patients undergoing matched unrelated donor BMT with our protocol (Dalal et al. 2000).

There have been few studies and reviews that have questioned the benefit of strict isolation for patients undergoing HSCT (Centers for Disease Control and Prevention; Infectious Disease Society of America; and American Society of Blood and Marrow Transplantation 2000; Sullivan et al. 2001; Yokoe et al. 2009). A recent survey found that even among patients with communicable diseases undergoing HSCT that isolation precautions were not used at many centres (Hicheri et al. 2013). However, careful analysis of these studies and reviews suggests that the lack of advantage from isolation was based on a lack of survival advantage for adult patients with malignancy undergoing HSCT, and the literature did not look at infectious morbidity. In adults, at least $85 \%$ of infections are caused by reactivation of the infection in the host rather than by acquired pathogens (van Kraaij et al. 2002). In contrast, for infants, acquired infections such as EBV, CMV, adenovirus, and varicella are usually transferred to the infant from their caregivers, rather than being host in origin. This factor suggests that more vigilant control of infectious exposure is required for infants, particularly for those with profound cellular immunodeficiencies.

The survival of cancer patients undergoing HSCT is impacted by factors such as disease recurrence and treatment-related morbidity, but these factors are not relevant for patients with nonmalignant diseases. A large study in aplastic anemia, using an isolation protocol similar to ours, demonstrated the importance of the use of a protective environment in reducing mortality (Storb et al. 1983). Isolation has also been shown to benefit HSCT patients by reducing the incidence of nosocomial respiratory viruses (Raad et al. 1997). The benefit of reverse isolation in protecting SCID patients from infections is best evidenced by David, who lived in a "bubble" for 12 years and remained free from severe infections (Guerra and Shearer 1986). It has also been 
shown that despite normal colonization of the gut and skin for an infant with SCID born by C-section and placed immediately in reverse isolation, the infant remained disease free for a 5-year period; this supports the importance of LAF and reverse isolation techniques in preventing significant infections in SCID patients (Taylor et al. 1978).

A protective environment has also been associated with delayed onset and reduced acute graft versus host disease (GVHD) frequency and severity, as infections may be a trigger for the development of GVHD (Storb et al. 1983). Preventing infections and immune stimulation is particularly important for SCID patients receiving HSCT, because of their increased risk for GVHD reported at $>70 \%$ in a large multicentre study (Grunebaum et al. 2006). This study found that GVHD and infections were the main cause of morbidity and mortality among these patients. The increased risk for severe and lethal GVHD is not surprising, as patients with immune deficiency have an abnormal/immature immune system with limited "negative selection" capacity (i.e., the ability to remove "auto-reactive" immune cells). Peripheral blood from SCID patients has revealed that they have markedly reduced levels of thymic dendritic and regulatory $\mathrm{T}$ cells (Treg), which are critical cells for immune regulation (Poliani et al. 2009; Somech et al. 2009). Treg are not expected to return to normal until after recovery of the thymus in patients with SCID, which occurs months after HSCT and may be even longer if prolonged immunosuppression is required to control GVHD (Cavazzana-Calvo et al. 2007).

The high financial cost of reverse isolation and LAF rooms has been used as an argument for not caring for SCID patients in this environment. A theoretical analysis of the nursing time spent preparing to enter patients' rooms ( 2 min hand scrub, mask, gown, and glove) in a 9-bed HSCT unit suggested an annual cost of USD \$7000/patient (Hayes-Lattin et al. 2005). However, when costs of providing isolation are offset against the financial cost of increased morbidity from GVHD and added in-patient hospital days the cost of reverse isolation is not so prohibitive.

It has also been argued that reverse isolation might have significant neurobehavioral effects on infants with SCID. Evidence from case reports of patients raised in reverse isolation for 28-52 months, and from David who lived in a bubble for 12 years, has shown no evidence of neurobehavioural problems in these children
(Freedman et al. 1976; Dalton 1981; Mueller 2011). From our unpublished observations of more than 25 years, we have not demonstrated impaired development, beyond that expected from the underlying disease, particularly when adequate social interventions and support are provided. However, the impact of the transplant experience on the development of young infants with SCID still requires further study.

Although studies assessing the effectiveness of a particular isolation protocol for SCID patients are lacking, it is also inappropriate to make deductions regarding required isolation for SCID patients from other patients who are secondarily immunosuppressed undergoing BMT. The unique immunological abnormalities and defective immune selection among patients with SCID supports the need to protect these patients from infections to reduce the morbidity and mortality from infections and GVHD. Our SCID reverse isolation protocol has been shown to have a low infection rate, and it has one of the best outcomes in the world for SCID patients undergoing HSCT.

\section{Acknowledgements}

Thanks to the nurses on the BMT/Immunology Isolation unit for their ongoing commitment to the SCID patients and their families.

\section{References}

American Association of Nurse Anesthetists. 2013. Infection control guide for certified registered nurse anesthetists [online]. Available from https://www. aana.com/resources2/professionalpractice/Documents/ PPM\%20Infection\%20Control\%20Guide.pdf [accessed 16 January 2015].

BMT Steering Committee. 2013. BMT4510/05 - Reverse isolation for BMT patients. SickKids policies \& proce dures. The Hospital for Sick Children, Toronto. Avail able from http://policies.sickkids.ca/published/Pages/ HomePage.aspx [accessed 16 January 2015].

BMT Steering Committee. 2014. BMT4540/06 - Preven tative maintenance on BMT in-patient unit $8 \mathrm{~B}$. Sick Kids policies \& procedures. The Hospital for Sick Children, Toronto. Available from http://policies.sick kids.ca/published/Pages/HomePage.aspx [accessed 16 January 2015].

Castro, B.A. 2008. The immunocompromised pediatric patient and surgery. Best Pract. Res. Clin. Anaesthesiol. 22(3):611-626. PMID: 18831306. 
Cavazzana-Calvo, M., Carlier, F., Le Deist, F., Morillon, E., Taupin, P., Gautier, D., Radford-Weiss, I., CaillatZucman, S., Neven, B., Blanche, S., Cheynier, R., Fischer, A., and Hacein-Bey-Abina, S. 2007. Longterm $\mathrm{T}$-cell reconstitution after hematopoietic stemcell transplantation in primary T-cell-immunodefi cient patients is associated with myeloid chimerism and possibly the primary disease phenotype. Blood. 109(10):4575-4581. PMID: 17272510. doi: 10.1182/ blood-2006-07-029090.

Centers for Disease Control and Prevention. 2014. Healthcare Infection Control Practices Advisory Committee (HICPAC) [online]. Available from http://www.cdc.gov/hicpac/pubs.html [accessed 7 October 2014].

Centers for Disease Control and Prevention; Infectious Disease Society of America; and American Society of Blood and Marrow Transplantation. (2000). Guidelines for preventing opportunistic infections among hematopoietic stem cell transplant recipients. MMWR. Recomm. Rep. 49(RR-10):1-125. PMID: 11718124.

Dalal, I., Reid, B., Doyle, J., Freedman, M., Calderwood, S., Saunders, F., and Roifman, C.M. 2000. Matched unrelated bone marrow transplantation for combined immunodeficiency. Bone Marrow Transplant. 25(6): 613-621. PMID: 10734295. doi: 10.1038/sj.bmt. 1702215 .

Dalton, R. 1981. The assessment and enhancement of development of a child being raised in reverse isolation. J. Am. Acad. Child Psychiatry. 20(3):611-622. PMID: 7310025.

Freedman, D.A., Montgomery, J.R., Wilson, R., Bealmear, P.M., and South, M.A. 1976. Further observations on the effect of reverse isolation from birth on cognitive and affective development. J. Am. Acad. Child Psychiatry. 15(4):593-603. PMID: 993485.

Gaspar, H.B., Qasim, W., Davies, E.G., Rao, K., Amrolia, P.J., and Veys, P. 2013. How I treat severe combined immunodeficiency. Blood. 122(23): 3749-3758. PMID: 24113871. doi: 10.1182/blood2013-02-380105.

Grunebaum, E., Mazzolari, E., Porta, F., Dallera, D., Atkinson, A., Reid, B., Notarangelo, L.D., and Roifman, C.M. 2006. Bone marrow transplantation for severe combined immune deficiency. JAMA. 295(5): 508-518. PMID: 16449616. doi: 10.1001/jama. 295.5.508.

Guerra, I.C., and Shearer, W.T. 1986. Environmental control in management of immunodeficient patients: Experience with "David". Clin. Immunol. Immunopathol. 40(1):128-135. PMID: 3521965.
Hayes-Lattin, B., Leis, J.F., and Maziarz, R.T. 2005. Isolation in the allogeneic transplant environment: How protective is it?. Bone Marrow Transplant. 36(5):373-381. PMID: 15968294. doi: 10.1038/sj. bmt.1705040.

Hicheri, Y., Einsele, H., Martino, R., Cesaro, S., Ljungman, P., and Cordonnier, C. 2013. Environmental prevention of infection in stem cell transplant recipi ents: A survey of the Infectious Diseases Working Party of the European Group for Blood and Marrow Transplantation. Transpl. Infect. Dis. 15(3):251-258. PMID: 23465046. doi: 10.1111/tid.12064.

Hubble, M.J., Weale, A.E., Perez, J.V., Bowker, K.E., MacGowan, A.P., and Bannister, G.C. 1996. Clothing in laminar-flow operating theatres. J. Hosp. Infect. 32(1):1-7. PMID: 8904367.

Mueller, A.T. 2011. Hospital helps recall story of 'Bubble Boy,' who would have been 40 this week. Available from http://healthcarecommunication.com/Main/Arti cles/Hospital_helps_recall_story_of_Bubble_Boy_who_ woul_7574.aspx [accessed 1 April 2014].

Patient Care Committee. 2010. Patient visiting. SickKids policies \& procedures. The Hospital for Sick Children, Toronto. Available from http://policies.sickkids.ca/ published/Pages/HomePage.aspx [accessed 16 January 2015].

Poliani, P.L., Facchetti, F., Ravanini, M., Gennery, A.R., Villa, A., Roifman, C.M., and Notarangelo, L. D. 2009. Early defects in human T-cell development severely affect distribution and maturation of thymic stromal cells: Possible implications for the pathophysiology of Omenn syndrome. Blood. 114(1): 105-108. PMID: 19414857. doi: 10.1182/blood-200903-211029.

Raad, I., Abbas, J., and Whimbey, E. 1997. Infection control of nosocomial respiratory viral disease in the immunocompromised host. Am. J. Med. 102 (3A):48-52. discussion 53-44. PMID: 10868143.

Smith, E.B., Raphael, I.J., Maltenfort, M.G., Honsawek, S., Dolan, K., and Younkins, E.A. 2013. The effect of laminar air flow and door openings on operating room contamination. J. Arthroplasty. 28(9):14821485. PMID: 23890828. doi: 10.1016/j.arth.2013. 06.012

Somech, R., Simon, A.J., Lev, A., Dalal, I., Spirer, Z., Goldstein, I., Nagar, M., Amariglio, N., Rechavi, G., and Roifman, C.M. 2009. Reduced central tolerance in Omenn syndrome leads to immature self-reactive oligoclonal $\mathrm{T}$ cells. J. Allergy Clin. Immunol. 124(4):793-800. PMID: 19767069. doi: 10.1016/j. jaci.2009.06.048. 
Storb, R., Prentice, R.L., Buckner, C.D., Clift, R.A., Appelbaum, F., Deeg, J., Doney, K., Hansen, J.A., Mason, M., Sanders, J.E., Singer, J., Sullivan, K.M., Witherspoon, R.P., and Thomas, E.D. 1983. Graftversus-host disease and survival in patients with aplastic anemia treated by marrow grafts from HLA-identical siblings. Beneficial effect of a protective environment. N. Engl. J. Med. 308(6):302-307. PMID: 6337323. doi: 10.1056/NEJM198302103080602.

Sullivan, K.M., Dykewicz, C.A., Longworth, D.L., Boeckh, M., Baden, L.R., Rubin, R.H., and Sepkowitz, K.A., Centers for Disease Control and Prevention; Infectious Diseases Society of America; and American Society for Blood and Marrow Transplantation Practice Guidelines and beyond. 2001. Preventing opportunistic infections after hematopoietic stem cell transplantation: The Centers for Disease Control and Prevention, Infectious Diseases Society of America, and American Society for Blood and Marrow Transplantation Practice Guidelines and beyond. Hematology Am. Soc. Hematol. Educ. Program. 392-421. PMID: 11722995.

Taylor, G.R., Kropp, K.D., and Molina, T.C. 1978. Microflora analysis of a child with severe combined immune deficiency. Infect. Immun. 19(2):385-390. PMID: 631877.

van Kraaij, M.G., Verdonck, L.F., Rozenberg-Arska, M., and Dekker, A.W. 2002. Early infections in adults undergoing matched related and matched unrelated/ mismatched donor stem cell transplantation: A comparison of incidence. Bone Marrow Transplant. 30(5): 303-309. PMID: 12209352. doi: 10.1038/sj.bmt.1703 643.

Yokoe, D., Casper, C., Dubberke, E., Lee, G., Munoz, P., Palmore, T., Sepkowitz, K., Young, J.A., and Donnelly, J.P. 2009. Infection prevention and control in healthcare facilities in which hematopoietic cell transplant recipients are treated. Bone Marrow Transplant. 44(8):495-507. PMID: 19861984. doi: 10.1038/bmt. 2009.261. 